\title{
FABRICATION OF SIZE-TUNABLE SILICA PARTICLES DURING SEED-GROWTH PROCESS
}

\author{
MACIEJ JARZĘBSKI ${ }^{\mathrm{a}, \mathrm{b}}$, TOMASZ ŚLIWA ${ }^{\mathrm{b}}$, MALGORZATA JARZĘBSKA, \\ KOSMA SZUTKOWSKI ${ }^{\mathrm{a}, \mathrm{c}}$

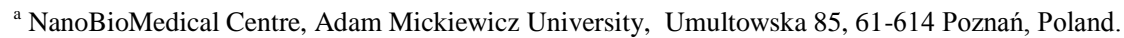 \\ ${ }^{\mathrm{b}}$ Molecular Biophysics Division, Faculty of Physics, Adam Mickiewicz University, Umultowska 85, 61-614 Poznan, Poland \\ ${ }^{c}$ Macromolecular Physics Department, Faculty of Physics, Adam Mickiewicz University, Umultowska 85, 61-614 Poznan, Poland
}

Received February 27, 2014; accepted March 25, 2014; published online March 28, 2014

\begin{abstract}
The size control is one of the most important factors during seed-growth process. Although precise determination of ingredients concentrations is easily controllable during the growth process, there is still a possibility to synthesize oversized particles. Nanosized fluorescent silica particles were synthetized using Stöber process and verified using three complementary methods of particle size determination, namely Dynamic Light Scattering (DLS), Particle Tracking (PT) as well as by Transmission Electron Microscopy (TEM) and Confocal Microscopy. The final diameter was verified by DLS and estimated to $494 \mathrm{~nm}$. Parameters necessary to control the size were derived.
\end{abstract}

\section{INTRODUCTION}

Silica microparticles and nanoparticles are one of the most widely used systems for variety of industrial and more recently for biomedical applications. In particular, the silicas are often used as simple raw materials or as fillers for polymer nanocomposites. Furthermore they can be used as pigment additives, as toothpaste additives and more recently as potential carriers for drug delivery systems. Accordingly, much of research work is focused on dispersion control and tailored surface modifications or synthesis of fluorescent beads and so-called hybrid structures. For example, Jesionowski and Krysztafkiewicz fabricated highly dispersed silica with glycerin introduced into the precipitation process. Their results showed partial blocking of the surface silica hydroxyl groups (silanol groups) and reduction of hydrophilicity (Jesionowski \& Krysztafkiewicz, 2002). Apart from difficulties related to dispersion of particles in the solution, equally important and needed property of silica particles is the surface functionalization so that desired property such as surface modification with fluorescent agents is achievable. In particular, the surface modification with aminosilane in methanol/water mixture results in formation of stable chemical bonds between the surface and dyes such as Reactive Blue 19 and Acid Violet 1 (Krysztafkiewicz, Binkowski \& Jesionowski, 2002).

The method described by Stöber et al. of controlled growth of monodisperse silica spheres in the micron size range (Stöber, Fink \& Bohn, 1968) has been modified many times to obtain desired nanosize or microsize particles. For instance, Canton et al. synthesized highly luminescent dye-doped silica nanoparticles with a wide the range of diameters $10-300 \mathrm{~nm}$. In addition, they determined experimental conditions under which bright monodisperse silica beads are obtained. The results were verified by Scanning Electron Microscopy (SEM). In particular, this kind of modified $\mathrm{SiO}_{2}$ particles are promising functional materials for bioimaging and biodetection applications acting as contrast agents and biosensors (Canton Ricco, Marinello, Carmignato \& Enrichi 2011).

Moreover, various types of functionalization protocols can be made as concerning silica nanosized particles so that desired properties, suitable for potential medical applications, are achieved. For instance, Liu et al. (Liu, Miyoshi \& Nakamura, 2007) prepared the core-shell gold-silica nanoparticles with thin mesoporous shell structures as it was previously described by Liz-Marzan et al. (Liz-Marzan, Giersig \& Mulvaney, 1996). These hollow mesoporous silica nanocapsules (HMSNs) loaded with fluorescein isothiocyanate (FITC) can be further used as a model system for controlled drug release systems. Other kind of modification of the HMSNs surface involves an addition of reactive groups which can enhanced bonding significantly with medically relevant macromolecules such as ligands and antibodies beeing on the outside of the silica particles. Rejeeth et al. attached cisplatin, to silica nanoparticles 
with size range varying from from 20 to $90 \mathrm{~nm}$ using aminopropyltriethoxy silane as a linker molecule where cisplatin is widely used chemotherapy drug. Their study showed the relationship between cisplatin release rate and the surface-to-volume ratio of the carrier beads (Rejeeth, Nag \& Kannan, 2013). Consequently, Irvine reported that nanoporous silica spheres wrapped around with fluid-phase lipid membranes are promising drug delivery systems for tumor treatment (Irvine, 2011). Although, for more efficient cancer therapy, so-called anticancer drug cocktails are being taken by patients (Vijayakameswara, 2013). Moreover, it turned out that the new particle was an effective cargo-carrying molecular shuttle for mix of doxorubicine and two other potential chemotherapeutics such as previously described cisplatin and 5-fluorouracil (Irvine, 2011).

In conclusion, the most important properties of particles, particularly in the nano range, are strongly influenced by size (Bell, Minelli, Tompkins, Stevens \& Shard, 2012). For that reason, the control of the particle size and distribution is crucial for many biomedical applications. Therefore the emphasis is put on the control of seed-mediated growth of the silica particles in the study.

It is well known that slight change in synthesis conditions impose on the size distribution and creates unrepeatable results. Though, the challenge is to synthesize the beads with a uniform size distribution In our case, the progress of the growth of silica particles was controlled by means of DLS, TEM as well as particle tracking technique. What is more, advantages and disadvantages of each method were thoroughly considered by Bell et al. (Bell et al., 2012).

Main goal of our study was the synthesis of fluorescent silica particles with hydrodynamic radii of around $200 \mathrm{~nm}$. Finally, our paper focuses on what valuable and useful information, during tailored particle synthesis, can be obtained from each of techniques. In order to achieve the desired control of the seed growth process DLS, TEM and PT techniques were used to monitor hydrodynamic radius as well as the size distribution.

\section{MATERIALS AND METHODS}

\section{Materials and sample preparation}

Fluorescent silica particles were synthesized from $98 \%$ tetramethyl orthosilicate (TEOS) obtained from Fluka, 29\% ammonium hydroxide (depicted in text as ammonia,) obtained from Sigma Aldrich, 99.8\% ethanol obtained from Bernd Kraft and millipored water. Rhodamine B isothiocyanate (RBITC), anhydrous 99.8\% methanol from Sigma Aldrich and 3aminopropyltriethoxysiliane (APS) from Fluka were used to synthesize the fluorescent labeling agent. The synthesis procedure is described by Verhaegh and van Blaaderen (Verhaegh N. A. M. \& van Blaaderen A., 1994), and with minor changes adapted in this paper. In particular, the synthesis procedure is divided into three steps: (1) preparation of the fluorescent agent, (2) core preparation, (3) seed growth and control.

Silica particles were doped with fluorescent dye Rhodamine B isothiocyanate. The dye was prepared using $0.1188 \mathrm{~g}$ of RBITC dissolved in $10 \mathrm{~mL}$ methanol (in Verhaegh N. A. M. \& van Blaaderen A., 1994, ethanol was used) and mixed with $0.099 \mathrm{ml}$ of APS $\left(\right.$ density $\left.=0.949 \mathrm{~g} \cdot \mathrm{mL}^{-1}\right)$. The sample was prepared in the glovebox with the inert atmosphere of nitrogen. The obtained solution of a dye was stirred for overnight at room temperature.

Silica cores were prepared in glass flask with flat bottom. Firstly Stöber mixtures were prepared by mixing $670 \mathrm{~mL}$ ethanol with $44 \mathrm{~mL}$ ammonia and $7 \mathrm{~mL}$ of water. Then $28.3 \mathrm{~mL}$ of TEOS was added to the mixture using glass funnel as recommended by Verhaegh N. A. M. \& van Blaaderen A., (1994). Afterward, the mixture was mixed with the fluorescent dye solution. Finally, dispersed particles were stirred for 18 hours. After stirring $0.7 \mathrm{~mL}$ of TEOS was added and then additional 6 parts of $5 \mathrm{~mL}$ of TEOS $(5 \mathrm{~mL}$ for 1 hour). Next, $10 \mathrm{~mL}$ of TEOS was filled up to a total amount of $253.7 \mathrm{~mL}$ of TEOS. The stirring speed was increased for 1 minute during addition. The seed growth process was monitored by DLS, TEM and particle tracking experiments.

Samples for DLS experiments. For DLS experiments, we took core samples after $5 \frac{1}{2}$ and 18 hours from the beginning of synthesis. Then we took coreshell samples (after TEOS addition) with 2, 7, 13, 23 and 30 shells. Each time the procedure was same as follow: $10 \mu \mathrm{L}$ of silica solution was dispersed in $2 \mathrm{~mL}$ of millipored water and vigorously stirred for 1 minute. Then the mixture was filtered through $5 \mu \mathrm{m}$ syringe filter to the measurement glass cuvette. The measurements on DLS was carried for 300s.

The mixture of silica cores and core-shell structures were stable and turbid. After addition of the whole volume of TEOS prepared, the seed-growth process ended while the supernatant solution remained pink. This confirms previous observations that not all RBITC molecules are bound to silica particles remaining in the solute phase explaining pink color (Verhaegh N. A. M. $\&$ van Blaaderen A., 1994). For PT measurements RBITC was removed by centrifugation with ethanol solution and then water.

\section{Particle characterization methods}

Dynamic Light Scattering (DLS). The hydrodynamic radii of particles $R_{h}$ were obtained by DLS. The measurements were performed using setup from ALVLaservertriebsgesellschaft (Langen, Germany) equipped 
with a Helium-Neon laser $(632.8 \mathrm{~nm})$ at $20^{\circ} \mathrm{C}\left( \pm 0.1^{\circ} \mathrm{C}\right)$. The scattering autocorrelation functions were obtained for scattering angles varied between $30^{\circ}$ and $150^{\circ}$ with the angular step $15^{\circ}$. The diffusion coefficients were obtained directly from the autocorrelation functions by using CONTIN algorithm for multi-exponential decay analysis (Provencher S.W., 1982). For a known diffusion coefficient, the hydrodynamic radius is obtained using the Stokes - Einstein relation:

$$
D=\frac{k_{B} T}{6 \pi \eta R_{h}},
$$

where $R_{h}$ is the hydrodynamic radius, $k_{B}$ is the Boltzmann constant, $T$ the absolute temperature, $\eta$ the solvent viscosity and $\mathrm{D}$ is diffusion coefficient. More thorough details on DLS experiment are described in detail by Berne and Pecora (Berne \& Pecora,1976).

An average diffusion coefficient obtained from DLS data is given by the equation:

$$
D_{D L S}=\frac{\sum D_{i} M_{i}^{2} N_{i}}{\sum M_{i}^{2} N_{i}}
$$

where $D_{i}, M_{i}, N_{i}$ are diffusion coefficients, molar weights and concentrations respectively.

In general, the intensity of the light scattering signal in the detector is proportional to the molecular mass and particle hydrodynamic radius. Although DLS is dustsensitive and post processing of data is limited, it is still rapid and robust method for quite precise particle characterization at relatively low costs. In spite of minor possibility of sample destruction during experiments, one can still operate on the same sample after sufficient period of time (Bell et al., 2012).

Particle Tracking (PT). The Brownian motion of silica particles was characterized using NanoSight NS500 with laser beam wavelength of $405 \mathrm{~nm}$. The particle size distribution was obtained. Conversely to DLS technique, the NanoSight NS500 instrument records videos for a whole population of particles undergoing Brownian motions in liquid solution. The particles are illuminated by laser light (NanoSight technical data 2012a). Subsequently, the images was collected using CCD camera and sufficient trajectories are selected e.g. whose lifetimes are long enough. The diffusive paths of the particles were measured in time span ranging from 10 to 90 s. Typical image frame used during analysis is presented in Figure 1. The diffusion coefficient is calculated from the recorded particle trajectory where mean square displacement is determined. Accordingly, the hydrodynamic radius is automatically determined from Stokes-Einstein equation for a known solvent viscosity and temperature (Eq. 1).
In the results we can obtain mean diameter (or radius) from distribution $\left(\mathrm{R}_{\mathrm{PTM}}\right)$ and mean from the peak maximum $\left(\mathrm{R}_{\mathrm{PTP}}\right)$.

The average diffusion coefficient is independent on the molar concentration of components and is given by Eq.3.

$$
D_{P T}=\frac{\sum D_{i} N_{i}}{\sum N_{i}}
$$

During particle tracking experiment, each sample was diluted in millipored water and temperature was kept at $20{ }^{\circ} \mathrm{C}$.

Transmission Electron Microscopy (TEM) and Laser Scanning Microscopy (LSM). The shape, size and morphology of the silica beads were examined by TEM, Zeiss Libra 120 at $120 \mathrm{kV}$. The silica particles were dispersed in $1 \%$ ethanol and placed on copper grids. The particle size distribution from TEM was calculated using ImageJ software and the radii was name $\mathrm{R}_{\mathrm{TEM}}$. Fluorescent behavior of particles was studied by confocal microscopy using Zeiss Laser Scanning Microscope LSM780 and Olympus IX71. During preparation, one droplet was taken from the sample and put onto microscopic glass and dry for few hours at 60 ${ }^{\circ} \mathrm{C}$. The beads were also studied in liquid solution. Prior to that, the slide channels, $1 \mu$ Slide from Ibidi or chamber cover glass 8 WELL from LAB-TEK, were filled up with the solution of fluorescent silica particles.

\section{RESULTS AND DISCUSSION}

DLS measurements. The example of DLS curves for selected silica particles with and without TEOS shells are shown in Fig. 1. The autocorrelation function is characterized by different correlation times therefore different values of diffusion coefficients and hydrodynamic radii are obtained. The results of DLS data analysis are summarized in Tab. 1. The hydrodynamic radii obtained from DLS data varied between 113 and $247 \mathrm{~nm}$ for silica cores and silica cores with 30 shells. What is more, there is minor difference between the size of a 'raw' core and the core with one shell or more due to relatively low volume of TEOS. For the sake of comparison between methods in use, some of DLS data is also reported in Tab. 2.

Particle size distribution of the nanoparticles can be obtained from the DLS correlation using CONTIN. Some larger differences between particle radii were obtained above $7^{\text {th }}$ shell but, in conclusion, each shell contributes to an average increase of hydrodynamic radius by 3-4 $\mathrm{nm}$. On the whole, the synthesis was stopped when the particle diameters reached desired size of around 400-500 nm. 


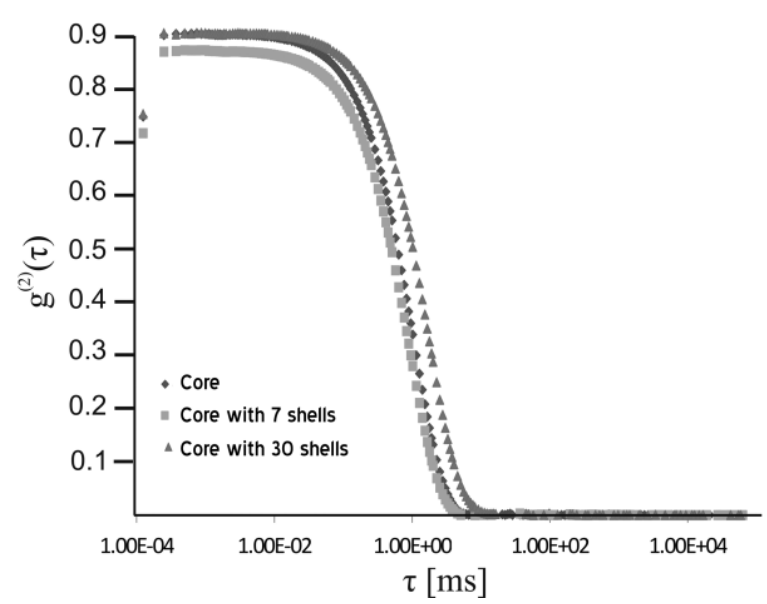

Fig. 1 Typical DLS curves of silica particles (from left core with 7 shells, core and core with 30 shells) measured at angle $90^{\circ}$.

Table 1.Hydrodynamic radii obtained by DLS

\begin{tabular}{lc} 
Sample description & $\begin{array}{c}\text { Hydrodynamic radius } \mathrm{R}_{\mathrm{h}} \\
{[\mathrm{nm}]}\end{array}$ \\
\hline Silica Core & 113 \\
Silica Core with 1 shell & 116 \\
Silica Core with 2 shells & 117 \\
Silica Core with 7 shells & 135 \\
Silica Core with 13 shells & 178 \\
Silica Core with 23 shells & 221 \\
Silica Core with 30 shells & 247 \\
\hline
\end{tabular}

Table 2. Mean radii $\mathrm{R}_{\mathrm{hDLS}}, \mathrm{R}_{\mathrm{PTM}}, \mathrm{R}_{\mathrm{PTP}}$ and $\mathrm{R}_{\mathrm{TEM}}$, of silica particles as determined by DLS, PT and TEM.

\begin{tabular}{lcccc}
\hline Sample & $\begin{array}{c}\mathrm{R}_{\mathrm{hDLS}} \\
{[\mathrm{nm}]}\end{array}$ & $\begin{array}{c}\mathrm{R}_{\mathrm{PTM}} \\
{[\mathrm{nm}]}\end{array}$ & $\begin{array}{c}\mathrm{R}_{\mathrm{PTP}} \\
{[\mathrm{nm}]}\end{array}$ & $\begin{array}{c}\mathrm{R}_{\mathrm{TEM}} \\
{[\mathrm{nm}]}\end{array}$ \\
\hline $\begin{array}{l}\text { Silica core } \\
\text { Silica core } \\
\text { with } 7^{\text {th }}\end{array}$ & 113 & 109 & 96 & 72 \\
$\begin{array}{l}\text { shell } \\
\begin{array}{l}\text { Silica core } \\
\text { with 30 }\end{array}\end{array}$ & 135 & 119 & 119 & 96 \\
shell & 247 & 189 & 184 & 195 \\
\hline
\end{tabular}

Particle Tracking. Mean particle diameters were obtained from tracked particle diffusive paths. An example of particle tracking experiment is shown in Fig.2. Brownian motions are recorded and trajectories are derived prior to calculation of diffusion coefficients.

The size distribution obtained for silica cores without TEOS shell and for cores with 7 and 30 shells are shown in Fig. 3. The maximum of diameters for solution of silica cores reaches $192 \mathrm{~nm}$ and for the core-shell structures particle diameters reached 237 and $368 \mathrm{~nm}$ for 7 and 30 shells respectively. The software allows to obtain cumulative data for all particle diameters although one can notice some differences between shapes of particle diameter distribution curves (Fig. 3).

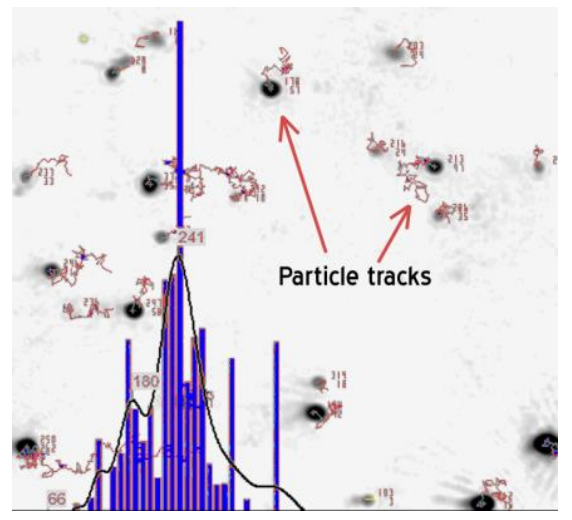

Fig. 2 Sample frame as obtained from NanoSight NS500 particle tracking instrument.

The PT results, along with DLS and TEM results, are summarized in Table 2. In the same way as for DLS experiment, obtained distributions of molecular sizes clearly indicate that the higher number of shells corresponds to higher molecular diameters. Contrary to DLS, particle sizes as obtained by PT, did not exceed $400 \mathrm{~nm}$ in diameter and $200 \mathrm{~nm}$ of radius. In other words, PT gives more accurate particle diameters, especially for higher number of shells. The deviation of the for DLS values reaches $30 \%$ in case of particles larger than $500 \mathrm{~nm}$. This might be the result of sample contamination or the way of how diffusion coefficients are determined by PT. The particle tracking method visualized the diffusive path of each particle being in the field of view. What is more, the dust is easily removed from the data. All in all, PT is slightly more accurate than DLS.

Nevertheless, our results indicate that for smaller particle sizes, DLS error is not exceeding 5\%. During PT method laser light illuminates particles where single particle is detected individually as the moving point of scattering undergoing Brownian motions. There is also possibility to use fluorescent mode if fluorescent particles are used which helps to identify and recognize selected (dyed) particles. The analysis software tracks and counts number of particles simultaneously (NanoSight - technical data 2012b). In our case the silica particles were dyed with mixed isomers of Rhodamine B isothiocyanate for which the maximum wavelength of absorption was $555 \mathrm{~nm}$ which was higher than the wavelength of the laser beam $(405 \mathrm{~nm})$. For that reason, the fluorescence signal was severely limited in amplitude and consequently mainly scatter mode was used during PT investigation. In conclusion, the fluorescence signal from the silica core with RBITC dye has to low intensity for the $405 \mathrm{~nm}$ excitation. However, some tests on fluorescent mode were performed (results not shown). One of the drawbacks of the PT method is the necessity of using larger sample volumes than for TEM. Although, PT can be used for determination of 

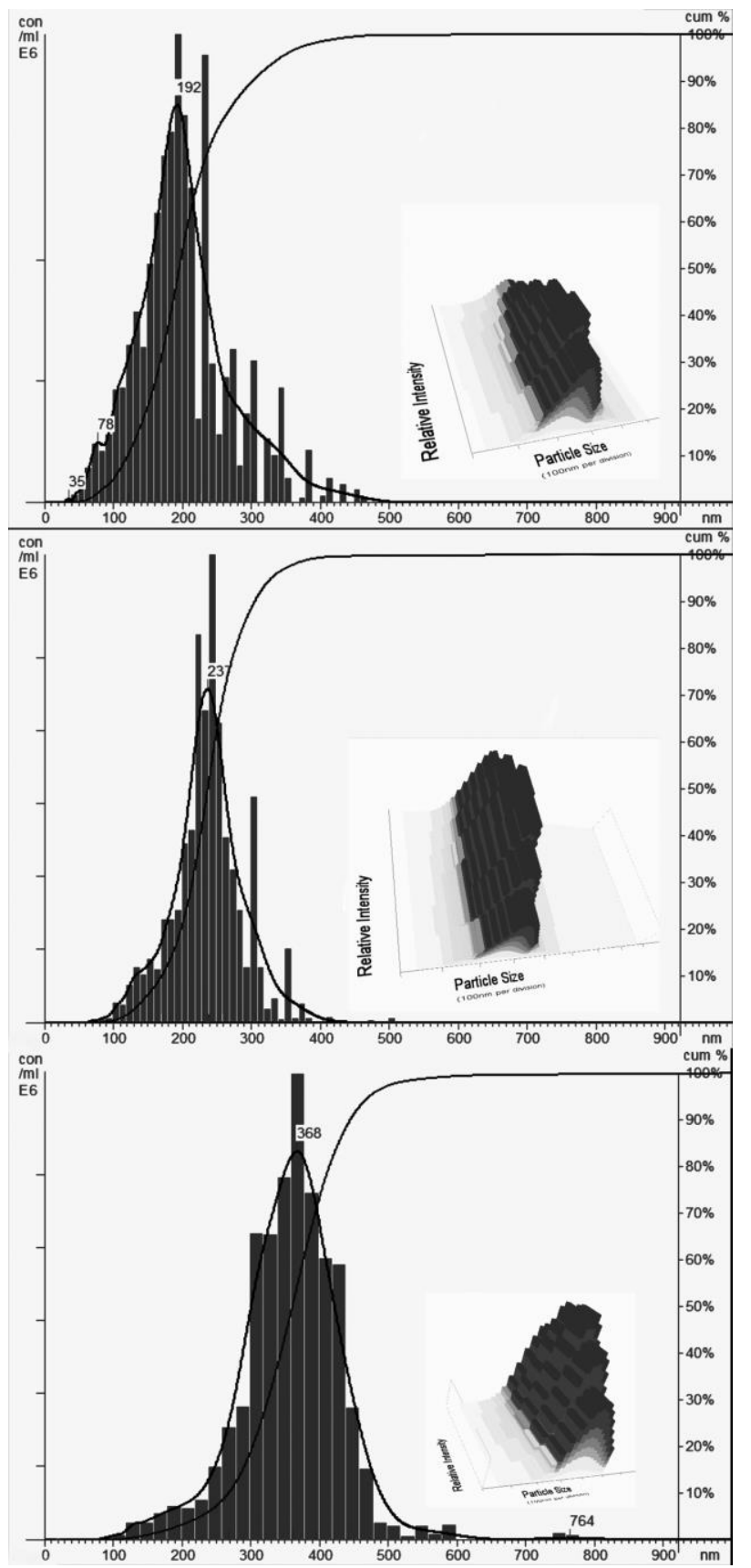

Fig. 3. Particle size distribution obtained by Particle Tracking method. Topmost: silica core, middle: silica core with 7 shells and bottommost: silica core with 30 shells.

concentrations in the sample. The PT system is analyzing particle size in the range $10-1000 \mathrm{~nm}$, while DLS can extend this value to both smaller and bigger particles with size ranging from 1 to $2000 \mathrm{~nm}$. Next, the PT operates particle-by-particle mode and does not analyze a whole population of particles as does DLS (Malloy, 2011).

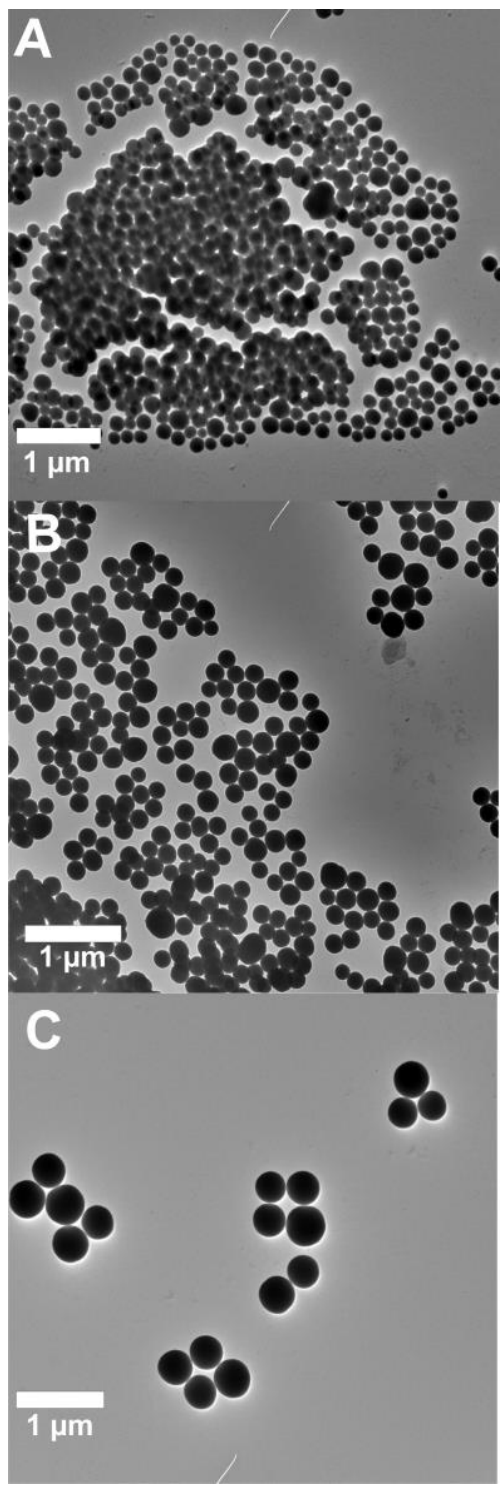

Fig. 4. TEM images obtained for fluorescent silica particles: (A) silica cores after 18 hours from synthesis, (B) silica cores with 7 shells, (C) final silica cores with 30 shells

TEM and LSM results. The shape and size of the nanoparticles was finally confirmed by TEM. The images of synthesized silica particles are presented in Fig. 4. The corresponding particle size distributions are presented in Fig.5. TEM results correspond well with data from PT. With the progress of the seed-growth process, the maximum of the diameter is shifting towards higher diameters, which is an indication of gradual growth of the particles.

All data is summarized in Tab. 2 which contains a comparison of the results as obtained by three methods, namely DLS, PT and TEM. The mean hydrodynamic diameters measured by PT are larger than those obtained 

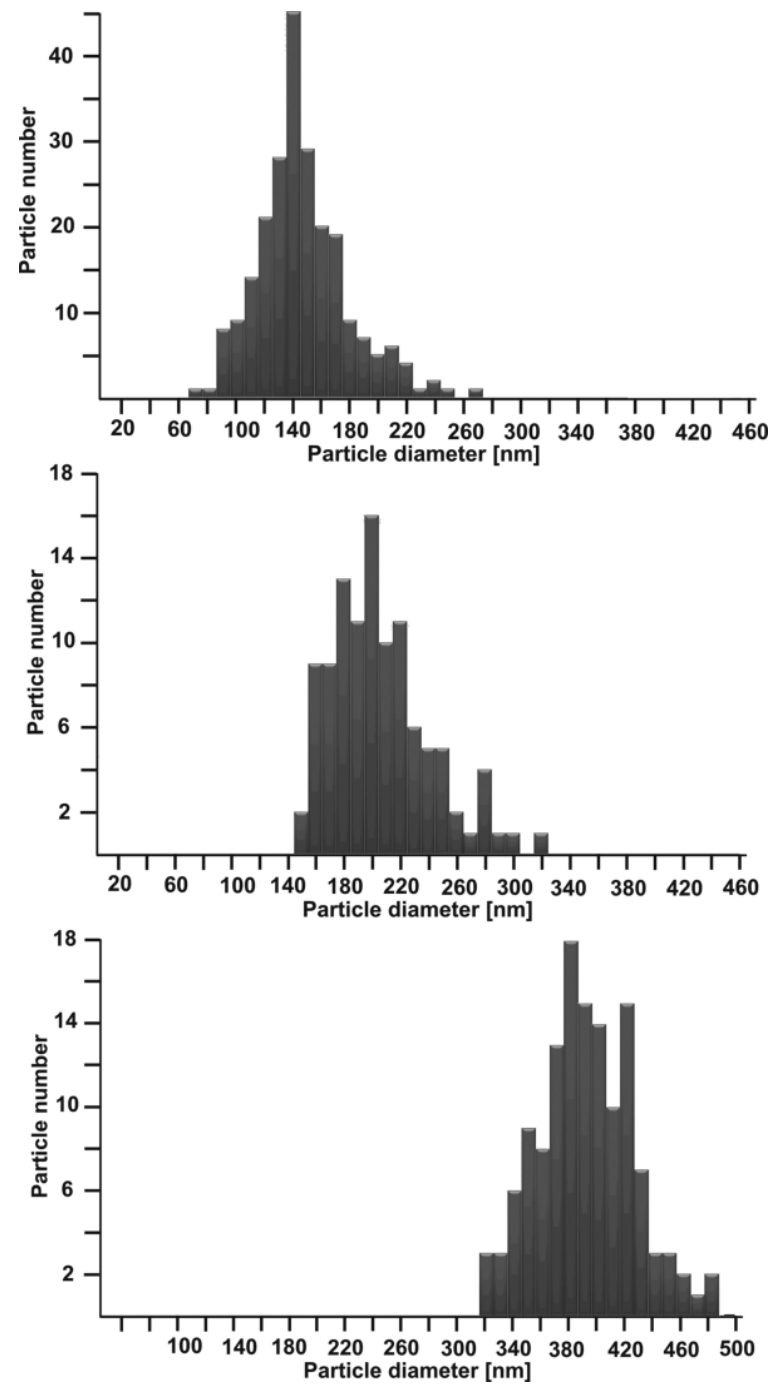

Fig. 5 Particle size distribution determined from TEM images. From bottom: silica cores, silica cores with 7 shells and silica cores with 30 shells.

by TEM. This result is consistent with previous investigations of silica beads (Bell et al., 2012; van Blaaderen A. \& Vrij A. 1992). Regardless the solvation effect, the $\mathrm{D}_{\mathrm{PT}}$ for largest particles is slightly lower than that observed by TEM. Considering sample preparation issues those results are in agreement.

LSM image for silica core with 30 shells is shown in Fig. 6. The LSM experiment was used to test fluorescence properties of obtained particles. Even for 30 shells the fluorescence did not vanish, which proves that particles can be further applied to bio-applications utilizing fluorescence.

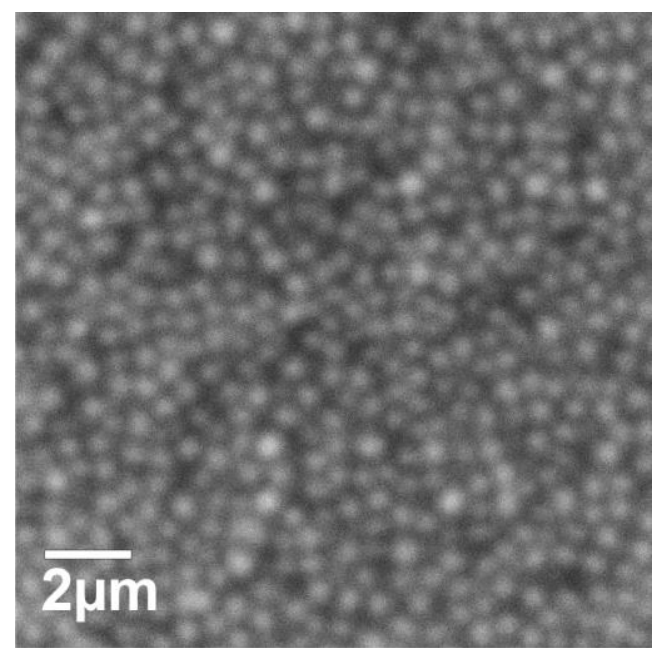

Fig. 6 Silica cores with 30 shells as obtained by Laser Scanning Microscope

\section{CONCLUSIONS}

Controlled silica particles with fluorescent-cores and transparent shells were synthesized using modified Stöber method. Particle size distribution was verified during synthesis using two laser techniques: DLS and PT. The advantages and disadvantages of each of the methods were briefly considered according to previous work (Bell et al., 2012). The disadvantages of TEM and LSM methods are relatively high costs and timeconsuming preparation process if compared to DLS and PT methods (Bell et al., 2012). Our investigations confirmed that for the final characterization of the silica particles it is important to use different techniques, although DLS and PT could be used for screening experiments. DLS and PT combined with TEM and LSM can characterize size and shape and optical properties of the material which is important for the purpose of studying synergy and interactions of particles with biological systems. All in all, DLS and PT are robust screening methods for control of the seed-growth. The sample preparation for TEM experiments is too complex and time consuming. Finally, one has to take into account that optical methods tend to overestimate particle diameters with respect to TEM results.

\section{ACKNOWLEDGMENTS}

Authors thanks to Johan Buitenhuis from Forschungszentrum Jülich, Institute of Complex Systems for help during synthesis and Adam Patkowski and Jacek Gapinski from Adam Mickiewicz University, Faculty of Physics for many fruitful discussions.

Maciej Jarzebski, thanks NanoBioMedical Centre in Poznan, and Faculty of Physics Adam Mickiewicz 
University that the work was supported by the International $\mathrm{PhD}$ Projects Programme of Foundation for Polish Science operated within the Innovative Economy Operational Programme (IE OP) 2007-2013 within European Regional Development Fund. Tomasz Sliwa thanks that Ph.D. thesis is carried under the project co-financed by European Union, program Human Capital National Cohesion Strategy founded by European Social Fund. Kosma Szutkowski gratefully acknowledge financial support from the National Centre for Research and Development under research grant "Nanomaterials and their application to biomedicine" PBS1/A9/13/2012.

\section{REFERENCES}

Bell N. C., Minelli C., Tompkins J., Stevens M. M. \& Shard A. G. (2012) Emerging Techniques for Submicrometer Particle Sizing Applied to Stöber Silica, Langumir, 28, 10860-10872.

Berne B.J., Pecora R., (1976) Dynamic Light Scattering: with Applications to Chemistry, Biology, and physics, Wiley, New York.

Bogush, G. H.; Tracy, M. A.; Zukoski, Iv, C. F. (1988) Preparation of Monodisperse Silica Particles: Control of Size and Mass Fraction. J. Non-Cryst. Solids, 104 (1), 95-106.

Bogush, G. H., Zukoski, Iv, C. F. (1991) Uniform Silica Particle Precipitation: An Aggregative Growth Model., J. Colloid Interface Sci., 142, 19 - 34.

Bogush, G. H., Zukoski, Iv, C. F. (1991) Studies of the Kinetics of the Precipitation of Uniform Silica Particles through the Hydrolysis and Condensation of Silicon Alkoxides., J. Colloid Interface Sci., 142, 1 - 18.

Canton G., Ricco R., Marinello F., Carmignato S., Enrichi F.,(2011) Modified Stöber synthesis of highly luminescent dye-doped silica nanoparticles., J. Nanopart. Res., 13, 43494356.

Irvine D. (2011) One particle, one kill, Nature Materials, 10, 342-343.

Jesionowski T., Krysztafkiewicz A. (2002) Preparation of the hydrophilic/hydrophobic silica particles., Colloids and Surfaces A: Physicochem. Eng. Aspects, 207, 49-58.

Krysztafkiewicz A., Binkowski S., Jesionowski T. (2002) Adsorption of dyes on a silica surface. Applied Surface Science, 199, 31-39.

Liu Y., Miyoshi H., Nakamura M. (2007), Novel drug delivery system of hollow mesoporous silica nanocapsules with thin shells: Preparation and fluorescein isothiocyanate (FITC) release kinetics, Colloids and Surfaces B: Biointerfaces 58, $180-187$.

Liz-Marzan L. M., Giersig M. \& and Mulvaney P. (1996), Synthesis of Nanosized Gold-Silica Core-Shell Particles, Langmuir, 12, 4329-4335.

Malloy A. (2011) Count, size and visualize nanoparticles, Materials Today, 14 (4), 170-173.

NanoSight (2012a) NTA 2.3 Analytical Software, Operating Manual P554J, Technical data, NanoSight Ltd., Minton Park, Amesbury, Wiltshire SP4 7RT, UK.
NanoSight (2012b) General Characterization Brochure Technical data, NanoSight Ltd., Minton Park, Amesbury, Wiltshire SP4 7RT, UK.

Provencher S.W. (1982). CONTIN: A general purpose constrained regularization program for inverting noisy linear algebraic and integral equations. Comput. Phys. Commun. 27, 229-232.

Vijayakameswara Rao N, Mutyala Naidu Ganivada, Santu Sarkar, Himadri Dinda, Koushik Chatterjee, Tanmoy Dalui, Jayasri Das Sarma, and Raja Shunmugam, (2013), Magnetic Norbornene Polymer as Multi-responsive Nanocarrier for Site Specific Cancer Therapy, Bioconjugate Chemistry. Just Accepted Manuscript, DOI: 10.1021/bc400409n.

Rejeeth, Nag \& Kannan (2013), Cisplatin-functionalized silica nanoparticles for cancer chemotherapy, Cancer Nano, $\mathbf{4}$, 127-136.

Stöber, W.; Fink, A.; Bohn, E. (1968) Controlled growth of monodisperse silica spheres in the micron size range. $J$. Colloid Interface Sci., 26 (1), 62-69.

van Blaaderen A. \&. Kentgens A.P.M (1992) Particle morphology and chemical microstructure of colloidal silica spheres made from alkoxysilanes. Journal of NonCrystalline Solids, 149, 161-178.

van Blaaderen A. \& Vrij A. (1992) Synthesis and Characterization of Colloidal Dispersions of Fluorescent, Monodisperse Silica Spheres. Langmuir, 8, 2921-2931.

van Blaaderen A. \& Vrij A. (1993) Synthesis and Characterization of Monodisperse Colloidal Organo-silica Spheres., J. Colloid Interface Sci., 256, 1-18.

Verhaegh N. A. M. \& van Blaaderen A. (1994) Dispersions of Rhodamine-Labeled Silica Spheres: Synthesis, Characterization, and Fluorescence Confocal Scanning Laser Microscopy., Langmuir, 10, 1427-1438.

Żurawska J., Krysztafkiewicz A. \& Jesionowski T. (2003) Physicochemical properties, surface morphology and particle size distribution of precipitated silicas., Surf. Interface Anal., 35, 914-921. 